\title{
On the Estimation and Properties of Logistic Regression Parameters
}

\author{
${ }^{1}$ Anthony Ngunyi, ${ }^{2}$ Peter Nyamuhanga Mwita, ${ }^{2}$ Romanus O. Odhiambo
}

\begin{abstract}
Logistic regression is widely used as a popular model for the analysis of binary data with the areas of applications including physical, biomedical and behavioral sciences. In this study, the logistic regression model, as well as the maximum likelihood procedure for the estimation of its parameters, are introduced in detail. The study has been necessited with the fact that authors looked at the simulation studies of the logistic models but did not test sensitivity of the normal plots. The fundamental assumption underlying classical results on the properties of MLE is that the stochastic law which determines the behaviour of the phenomenon investigated is known to lie within a specified parameter family of probability distribution (the model). This study focuses on investigating the asymptotic properties of maximum likelihood estimators for logistic regression models. More precisely, we show that the maximum likelihood estimators converge under conditions of fixed number of predictor variables to the real value of the parameters as the number of observations tends to infinity. We also show that the parameters estimates are normal in distribution by plotting the quantile plots and undertaking the Kolmogorov -Smirnov an the Shapiro-Wilks test for normality, where the result shows that the null hypothesis is to reject at $0.05 \%$ and conclude that parameters came from a normal distribution.
\end{abstract}

Key Words: Logistic, Asymptotic, Normality, MRA(Multiple Regression Analysis)

\section{Introduction}

Regression analysis is one of the most useful and the most frequently used statistical methods [24, 3]. The aim of the regression methods is to describe the relationship between a response variable and one or more explanatory variables. Among the different regression models, logistic regression plays a particular role. The basic concept, however, is universal. The linear regression model is, under certain conditions, in many circumstances a valuable tool for quantifying the effects of several explanatory variables on one dependent continuous variable. For situations where the dependent variable is qualitative, however, other methods have been developed. One of these is the logistic regression model, which specifically covers the case of a binary (dichotomous) response. [6] discussed an overview of the development of the logistic regression model. He identifies three sources that had a profound impact on the model: applied mathematics, experimental statistics, and economic theory. [?] also provided details of the development on logistic regression in different areas. He states that, "Sir [5] introduced many statisticians to logistic regression through his 1958 article and 1970 book, "The Analysis of Binary Data". However, logistic regression is widely used as a popular model for the analysis of binary data with the areas of applications including physical, biomedical, and behavioral sciences.

In this study, the logistic regression models, as well as the maximum likelihood procedure for the estimation of their parameters, are introduced in detail. Based on real data set, an attempt has been made to illustrate the application of the logistic regression model.

Simulation is used in the study since it involves construction of complicated integrals that do not exists in a closed form that can be evaluated. Simulation methods can be used to evaluate it to within acceptable degrees of approximation by estimating the expectation of the mean of a random sample.

\section{Literature Review}

The method of maximum likelihood is the estimation method used in the logistic regression models, however, two other methods have been and may still be used for estimating the coefficient . These methods are the least squares and the discriminant function analysis. The linear model approach of analysis of categorical data proposed by Grizzle et al.(1969) used estimaton based on NonLinear Weighted S(NLWS). They demostrated that logistic model can be handled by the method of maximum likelihood using an iterative reweighted least squares algorithm. The discriminant approach to estimation of the coefficients is of historical importance as popularized by [4]. [14] compared the two methods when the model is dichotomous and

\footnotetext{
${ }^{1}$ Dedan Kimathi University of Technology $($ Kenya $)$ P.O BOX 657-10100, Nyeri

${ }^{2}$ Jomo Kenyatta University of Agriculture \& Technology (Kenya)

P.O BOX 62000-00200, Nairobi
} 
concluded that the discriminat function was sensitive to the assumption of normality. In particular, the estimation of the coefficient for the nonnormal distributed variables are biased away from zero, when the coefficient is in fact different from zero. This implies that for the dichotomous independent variable the discrimnant function will overestimate the magnitude of the coefficient.

According to [13], the fact concerning the interpretability of the coefficients is the fundamental reason why logistic regression has proven such a powerful analytic tool for epidemiologic research. At least, this argumentation holds whenever the explanatory variables $x$ are quantitative. [9] investigate the asymptotic properties of various discrete and qualitative response models (including logit model) and provided conditions under which the MLE has its usual asymptotic properties, that is, the $p$-vector $\beta$ of coefficients of linear combinations $(x, \beta)$ has to be estimated from a finite sample of $n$ observations. The method of analysis of generalized linear models can be used since logistic models are sub-category [17].

[11] established that the maximum likelihood estimators are the best asympotically and strong consistent estimators of the logit model, other estimators have been suggested for logit model including the minimum $\phi$ divergent estimator which are generalization of maximum likelihood and are also consistent and asymptotically normal [20].

[25] discussed the inconsistency of the generalized method of moments estimator of qualitative models with random regressors and suggested a suitable modification in case of the probit and not the logit.

In the parameter estimation and inference in statistics, maximum likelihood has many optimal properties in estimation: sufficiency (complete information about the parameter of interest contained in its estimation); consistency (true parameter value that generated the data recovered asymptotically, i.e. data of sufficiently large samples); efficiency (lowest possible variance of parameter estimates achieved asymptotically) and parameterization invariance. The asymptotic normality of the maximum likelihood in logistic regression models are also found in [18] and [19]. [18] presents regularity conditions for a multinomial response model when the logit link is used. [19] presents regularity conditions that assure asymptotic normality for the logit link in binomial response models and further verifies that his conditions are equivalent to those of [18]. [7] discuss the asymptotic distribution of the MLE for constructing confidence intervals and conducting tests of hypotheses.[12] prove that the MLE is asymptotically normal in this setting as long as certain regularity conditions are satisfied

\subsection{Logistic function}

The function has been discussed by many reseachers like [10]. It is given by;

$$
\begin{aligned}
& f(g)=\frac{\exp (g)}{1+\exp (g)} \\
& =\frac{1}{1+\exp (g)}
\end{aligned}
$$

when modelling a bernoulli random variable with multivariables, one directly models the probabilities of group membership, as follows;

$$
P(Y=1 \mid X=x)=\frac{1}{1+\exp \left(-\left(\beta_{0}+\sum_{j=1}^{d} x_{i j} \beta_{j}\right)\right)}
$$

where $g$ in 1 is given by

$$
g(X ; \beta)=\beta_{0}+\beta_{1} X_{11}+\beta_{2} X_{12}+\cdots+\beta_{d} X_{1 d}
$$

To illustrate, the applicability of the logistic function, the bold curve in the figure 0 shows that the logistic function puts more weight on the tails than the normal distribution. 


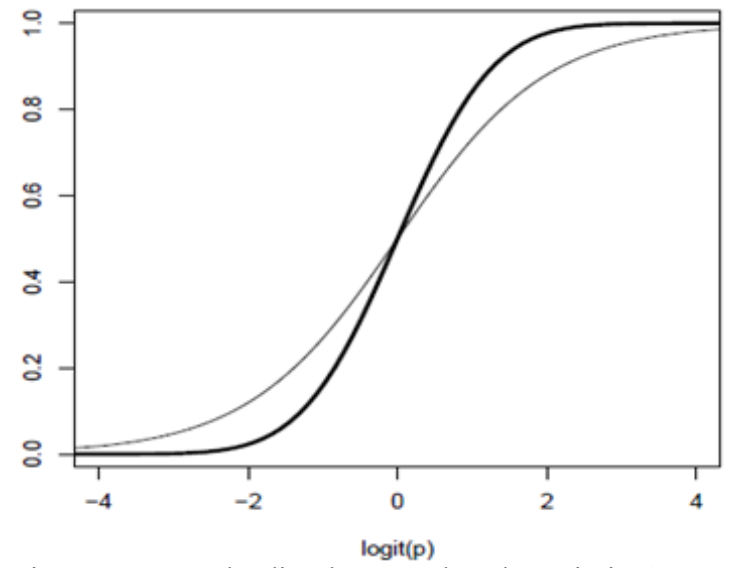

Figure 1: Standardized Normal and Logistic CDF's

The logistic model is bounded between zero and one, this property estimates the possibility of getting estimated or predicted probabilities outside this range which would not make sense. Also with a proper transformation, one can get a linear model from the logistic function. [10] uses the logit function of the Bernoulli distributed response variable. Transforming 2 as in [10] we have ;

$$
\begin{aligned}
& \operatorname{Logit}[P(Y=1 \mid X=x)]=\log _{e} \frac{P(Y=1 \mid X=x)}{1-P(Y=1 \mid X=x)} \\
& =\log _{e}\left\{\frac{1+\exp \left(\beta_{0}+\sum_{j=1}^{d} \beta_{j} X_{i j}\right)}{1+\exp \left(-\left(\beta_{0}+\sum_{j=1}^{d} \beta_{j} X_{i j}\right)\right)}\right\} \\
& =\log _{e}\left(\exp \left(\beta_{0}+\sum_{j=1}^{d} \beta_{j} X_{i j}\right)\right) \\
& =\beta_{0}+\sum_{j=1}^{d} \beta_{j} X_{i j}
\end{aligned}
$$

the function 4 is a generalized linear model (GLM) with $d$ independent variables.

The motivation to the use of logistic model is that it follows the properties of the GLM. Lets define the hypothetical population proportion of cells for which $Y=1$ as $\pi=P(Y=1 \mid X=x)$. Then the theoretical proportion of cells for which $Y=0$ is $1-\pi=P(Y=0 \mid X=x)$. We estimate $\pi$ by the sample proportions of cells for which $Y=1$. In the GLM context, it is assumed that there exists a set of predictor variables, $X_{11}, X_{12}, \cdots, X_{1 d}$, that are related to $Y$ and therefore provides additional information for estimating $Y$. For mathematical reasons of additivity and multiplicity, logistic model is based on linear model for the log odds in favour of $Y=1$.

$$
\log _{e} \frac{\pi_{i}}{1-\pi_{i}}=\alpha+\sum_{j=1}^{d} \beta_{j} X_{i j}
$$

thus

$$
\pi_{i}=\sum_{j=0}^{d} \beta_{j} X_{i j}
$$

where $\beta \in \mathfrak{R}^{d}$ of unknown parameters.

The logistic regression (logit link) 


$$
g\left(\pi_{i}\right)=\log _{e} \frac{\pi_{i}}{1-\pi_{i}}=\operatorname{logit}\left(\pi_{i}\right)
$$

and

$$
g^{-1}\left(g\left(\pi_{i}\right)\right)=\pi_{i}
$$

thus the inverse of the logit function in terms of $(X ; \beta)$ is given by;

$$
g^{-1}(X ; \beta)=\pi_{i}=\frac{1}{1+\exp \left(-\left(\beta_{0}+\sum_{j=1}^{d} x_{i j} \beta_{j}\right)\right)}
$$

This model can be rewritten as

$$
\operatorname{logit}\left(\pi_{i}\right)=\sum_{j=0}^{d} \beta_{j} X_{i j}
$$

\section{Methodology}

\subsection{Maximum Likelihood Estimation of the Parameter $\beta$}

[10] pointed out that estimating the function $P(Y=1 \mid X=x)$ in 1 is equivalent to estimating the function $g(X ; \beta)$ 2. Parametric estimation of $g(X ; \beta)$ can be found in [15], [21] and [22] among other authors, they used the maximum likelihood estimation method. As they pointed out, one first defines the likelihood function. For the Bernoulli distribution case we have

$$
L(Y, X ; \beta)=\prod_{i=1}^{n}[P(Y=1 \mid X=x)]^{y_{i}}[1-P(Y=1 \mid X=x)]^{1-y_{i}}
$$

So, taking the logarithm and upon simplification we have

$$
L(Y, X ; \beta)=\sum\left\{\left(y_{i}-g(X ; \beta)-\log _{e}(1+\exp (g(X ; \beta)))\right\}\right.
$$

The regularity conditions requires that the MLEs of $\beta$ satisfies the usual consistency and asymptotic normality properties $[1,11]$.

The optimization of the function in 5 with respect to the unknown vector $\beta$ requires iterative techniques since first derivative is nonlinear in $\hat{\beta}$ and has no simple analytical solution for $\hat{\beta}$ [16].

$$
\begin{aligned}
& L^{\prime}(Y, X ; \beta)=\sum_{i=1}^{d} y_{i} x_{i j}-n_{i} \frac{\exp \left(\sum_{i=1}^{d} \beta_{j} X_{i j}\right)}{1+\exp \left(\sum_{i=1}^{d} \beta_{j} X_{i j}\right)} x_{i j} \\
& =\sum_{i=1}^{d} y_{i} x_{i j}-n_{i} \pi_{i} x_{i j}
\end{aligned}
$$

In matrix form, 7 can be rewritten in the form;

$$
L^{\prime}(Y, X ; \beta)=\sum_{i=1}^{n}\left(y_{i}-\pi_{i}\right) \mathbf{X}
$$

The equation, 


$$
\pi_{i}=\frac{\exp \left(\sum_{i=1}^{d} \beta_{j} X_{i j}\right)}{1+\exp \left(\sum_{i=1}^{d} \beta_{j} X_{i j}\right)}
$$

is strictly increasing function (monotone) of $\beta_{j}$ and approaches 0 as $\beta_{j} \rightarrow \infty$ and approaches $n$ as $\beta_{j} \rightarrow \infty$. The second derivative of 6 is strictly negative for all $\beta_{j}{ }^{\prime} s$ and as such the solution is a maximum $[2,23]$.

\subsection{Numerical Optimization}

The Newton-Rapson method requires that the starting values be sufficiently close to the solution to ensure convergence. Under this condition the Newton-Raphson iteration converge quadratically to at least a local optimum. When the method is used to the problem of maximizing the likelihood function, it produces a squence of values $\theta^{(0)}, \theta^{(1)}, \cdots, \theta^{(\theta)}$ that under ideal conditions converge to the MLEs $\hat{\theta}_{\text {mle }}$.

the motivation to the use of the method is that this approximation is valid provided the unknown parameter $\beta^{j+1}$ is in the neighbourhood of $\beta^{j}$. Since $L(Y, X ; \beta)$ corresponds to the objective function to be minimized, $L^{\prime}(Y, X ; \beta)$ represents the gradient vector, the vector of first order partial derivative and $I(\theta)$ to the negative of the Hessian matrix $H(\theta)$ which is a matrix of the second order derivative of the objective function $L^{\prime \prime}(Y, X ; \beta)$. Then the Hessian matrix is used to determine whether the minimum of the objective function $L(Y, X ; \beta)$ is achieved by the solution $\hat{\theta}$ for the equation $L^{\prime}(Y, X ; \beta)=0$, that is, whether $\hat{\theta}$ is a stationary point of $L(Y, X ; \beta)$. If this is the case the $\hat{\theta}$ is the maximum likelihood estimate of the matrix of $\theta$ the iterative formula for finding a maximum or minimum of a function $f(x)$ is given by;

$$
X^{(j+1)}=X^{(j)}-H_{i}^{-1} l^{\prime}(\theta)
$$

where $H_{i}$ is the Hessian $f^{\prime \prime}\left(X_{i}^{j}\right)$ and $l^{\prime}(\theta)$ is the gradient vector, $f^{\prime}(x)$ of $f(x)$ at the $i^{\text {th }}$ iteration.

Then the $i^{\text {th }}$ iteration is given by;

$$
\hat{\theta}^{(j+1)}=\hat{\theta}^{(j)}-\left(H(\hat{\theta})^{j}\right)^{-1} l^{\prime}(\theta)
$$

In otherwords,

$$
\hat{\theta}^{(j+1)}=\hat{\theta}^{(j)}-\frac{l^{\prime}(\theta)}{l^{\prime \prime}(\theta)}
$$

which is the iterative generator.

But from 9

$$
L^{\prime}(Y, X ; \beta)=\sum_{i=1}^{d} y_{i} x_{i j}-n_{i} \pi_{i} x_{i j}
$$

In matrix form;

$$
L^{\prime}(Y, X ; \beta)=\sum_{i=1}^{n}\left(y_{i}-\pi_{i}\right) \mathbf{X}
$$

and the negative of the second derivative;

$$
I(\beta)=\frac{\partial^{2} L(Y, X ; \beta)}{\partial \beta \partial \beta^{\prime}}=\sum_{i=1}^{n} \pi_{i}\left(1-\pi_{i}\right) \mathbf{X}^{\prime} \mathbf{X}
$$

The matrix $I(\beta)$ is a $(p+1) \times(p+1)$ matrix. The matrix plays a key role in the estimation procedure and 
yields the logit estimates obtained by inverting the Hessian (or expected Hessian ) matrix or the information matrix. Then the Newton-Raphson iterative solution of the system of equations can be used to obtain the solution of $\beta^{\prime} s$. At the $i^{t h}$ iteration, estimates are obtained as;

$$
\hat{\beta}^{(i+1)}=\hat{\beta}^{(i)}-\left[I\left(\hat{\beta}^{i}\right)\right]^{-1} L^{\prime}\left(Y, X ; \hat{\beta}^{i}\right)
$$

where the least square estimates of the $\beta^{\prime}$ s are used as initial estimates.

Continue applying Equation 10 until there is essentially no change between the elements of $\beta$ from one iteration to the next. At that point, the maximum likelihood estimates are said to converge.

\section{Simulation study}

\subsection{Checking consistency of the maximum likelihood Estimators}

Nonlinear system of equations arise commonly in statistic. In some cases, there will be a naturally associated scalar function of parameters which can be optimized to obtain parameter estimates. The MLE cannot be written in closed form expression, thus substantialy complicating the task of evaluating the characteristic of its (finite sample) distribution, whether the variables are random or not. Maximum likelihood estimator simulation for large samples are carried out using the Monte-Carlo simulation method. The simulations of the study involves the regressor variables which are fixed and for each model parameter, $\mathrm{n}$-simulation binomial data set are generated for each of the regressor variable $x_{1}, x_{2}, \cdots, x_{n}$. We consider the complete model to be simulated as;

$$
\begin{aligned}
& y_{i}=g(X ; \beta)+e_{i} \\
& =1 \text { if } X_{i} \beta+e \geq a \\
& =0 \text { if } X_{i} \beta+e<a
\end{aligned}
$$

where $y_{i}$ is the dependent variable to incorporate the effects of the independent variables. The row vector $X_{i}$ represents the $i^{\text {th }}$ observations on all predictor variables.

The basic model can be structured as

$$
\begin{aligned}
& \pi_{i}=\operatorname{Pr}\left(y_{i}=1 \mid x_{i}\right) \\
& 1-\pi_{i}=\operatorname{Pr}\left(y_{i}=0 \mid x_{i}\right)
\end{aligned}
$$

For the logit model;

$$
\pi_{i}=\frac{\exp \left(x^{\prime} \beta\right)}{1+\exp \left(x^{\prime} \beta\right)}
$$

\begin{tabular}{|c|c|c|c|c|c|c|c|c|}
\hline \multirow{2}{*}{$\beta$} & \multicolumn{3}{|c|}{$n=200$} & $n=300$ & \multicolumn{3}{|c|}{$n=500$} & \multirow{2}{*}{$\begin{array}{c}n=700 \\
\text { SE }\end{array}$} \\
\hline & Estimates & SE & Estimates & SE & Estimates & $\mathrm{SE}$ & Estimates & \\
\hline$\beta_{0}$ & 42.356 & 472.855 & 24.268 & 12.830 & & $\begin{array}{r}-22.872 \\
3.583\end{array}$ & 22.497 & 2.947 \\
\hline$\beta_{1}$ & 6.237 & 73.156 & & $\begin{array}{l}3.425 \\
2.173\end{array}$ & & $\begin{array}{ll}3.236 & \\
0.436\end{array}$ & & 3.1770 .357 \\
\hline$\beta_{2}$ & 0.310 & 4.085 & & $\begin{array}{r}0.136 \\
0.079\end{array}$ & & $\begin{array}{ll}0.129 & \\
& 0.057\end{array}$ & & $\begin{array}{l}0.127 \\
0.047\end{array}$ \\
\hline$\beta_{3}$ & 0.039 & 0.716 & & $\begin{array}{r}0.036 \\
0.024\end{array}$ & & $\begin{array}{ll}0.034 & \\
& 0.018\end{array}$ & & $\begin{array}{r}0.033 \\
0.015\end{array}$ \\
\hline$\beta_{4}$ & 1.501 & 2.204 & & $\begin{array}{ll}0.983 & \\
0.29\end{array}$ & & $\begin{array}{ll}0.937 & \\
& 0.206\end{array}$ & & $\begin{array}{l}0.920 \\
0.169\end{array}$ \\
\hline
\end{tabular}

which is the cdf of the logistic distribution.

For each generated data set, the mle for $\hat{\beta}$ is computed and saved. This procedure is repeated for $n=200,300,500$, and 700 at each of the regressor levels.

The following table gives the results of the simulation study for different sample sizes.

Table 1: Estimated-parameter values and their standard errors using the regression model for different sample 
As seen in the table 0 , as the sample size increases from $n=200$ to $n=700$ the estimated values of the parameters are very close to the true values $\beta_{0}, \beta_{1}, \beta_{2}, \beta_{3}$, and $\beta_{4}$ and the standard deviations of the estimates are noticeably smaller. This indicates that this simulation study performs well in showing the consistency of the maximum likelihood estimators for parameters of the logistic model.

\subsection{Regularity conditions of the asymptotic normality of a Binomial Response model}

[9] present regularity conditions for a very general class of generalized linear models. In this section, we explain the regularity conditions under the Binomial response model and then we apply Theorem $\mathbf{1}$ to show the asymptotic properties of ML estimators for the Binomial response model.

(C1): The pdf $g(X ; \beta)$ is distinct, that is $\beta \neq \beta^{\prime}$ implying that $g(X ; \beta) \neq g\left(X ; \beta^{\prime}\right)$, thus the model is identifiable.

The proof of this assumption has been well documented by [23]

(C2): The pdf have common support for all $\beta$, the true parameter vector is in the interior of this space.

This condition holds if the domain (support) of $X$ is a closed set [18].

[18] noted that the restriction that true parameter vector in the interior excludes some cases where consistent and asymptotically normal (CAN) breaks down. This is not a restrictive assumption in most application, but it is for some.

(C3): The response model is measurable in $x$, and for almost all $x$ is continous in the parameters. The standard models such as the probit, logit and the linear probability model are all continous in their argument and in $x$, so that this assumption holds.

(C4): The model satisfies a global identification (that is it guarantees that there is at most one global maxima, see [18].

The proof of this assumption has been discussed well by [23]. The concavity of the log-likelihood of an observation for the logit guarantees global identification, provided only that the $x^{\prime} s$ are not linearly independent.

(C5): The assumption states that the model log likelihood is twice or three times differentiable, this is true provided the parameters do not give observations on the boundary in the linear or log linear models where probabilities are zero or one. [8] shows that these conditions are specifically satisfied for the binomial model.

(C6): The log likelihood and its derivative have bounds independent of the parameters in some neighbourhood of the true parameter values. The first derivative have the Lipschitz property in the neighbourhood. This property is satisfied by the logistic model since it is continously differentiable (McFadden,1999).

(C7): The pdf $g(X ; \beta)$ is three times differentiable as a function of $\beta$. Further, for all $\beta \in \Omega$, there exists a constant $c$ and a function $M(x)$ such that for all $\beta_{0}-c<\beta<\beta_{0}+c$ and all $x$ in the support of $X$.

$$
\left|\frac{\partial^{3}}{\partial \beta^{3}} \log g(X ; \beta)\right| \leq M(x)
$$

with

$$
E_{\beta_{0}}[M(X)]<\infty
$$

for all $\beta_{0}-c<\beta<\beta_{0}+c$ and all $x$ in the support of $X$. The proof of this assumption has been done by many authors like $[2,23]$. This implies that the information matrix, equal to the expectation of the outer product of the score of an observation is non-singular at the true parameter.

The conditions $(C 1), \cdots,(C 7)$ may seem restrictive at first, but are met for a wide range of link functions. The results guarantee that the MLE estimates of $\beta$ is essentially carried out by linearizing the first order condition for the estimator using a Taylor's expansion. Since the binomial model satisfies the above conditions, then following theorem holds for the parameter $\bar{\beta}$.

1 Let $x_{1}, x_{2}, x_{3}, \cdots, x_{n}$ be iid each with a density $g(x ; \beta)$. Then, with probability tending to 1 as 
$n \rightarrow \infty$, there exists solutions $\hat{\beta}_{n}=\hat{\beta}\left(x_{1}, \cdots, x_{n}\right)$ of the likelihood equations.

or equivalently

$$
\frac{\partial}{\partial \beta_{j}}\left[g\left(x_{1} ; \beta\right), \cdots g\left(x_{n} ; \beta\right)\right]=0, \quad j=1,2, \cdots, n
$$

such that

$$
\frac{\partial}{\partial \beta_{j}}[\log L(\beta)]=0, \quad j=1,2, \cdots, d
$$

(a) $\hat{\beta}_{j n}$ is consistent for estimating $\beta_{j}$.

(b) $\sqrt{n}\left(\hat{\beta}_{n}-\beta\right)$ is asymptotically normal with mean (vector) zero and covariance matrix $\left[L(\beta)^{-1}\right]$, and

(c) $\hat{\beta}_{j n}$ is asymptotically efficient in the sense that

$$
\sqrt{n}\left(\hat{\beta}_{j n}-\beta_{j}\right) \underline{L} N\left(0,[I(\beta)]_{j j}^{-1}\right)
$$

\subsection{Normality of the ML estimators}

Under some assumptions that allows among several analytical properties, the use of the delta method, the central limit theorem holds. We conducted a simulation study via the freeware package $\mathrm{R}$. We show how the properties of an estimator are affected by changing conditions such as its sample size and the value of the underlying parameters. Employing it in practice, we illustrate the large sample behavior of the estimated parameters $\hat{\beta}=\left(\hat{\beta}_{0}, \hat{\beta}_{1}, \hat{\beta}_{2}, \hat{\beta}_{3} \text {, and } \hat{\beta}_{4}\right)^{\prime}$ and also look at the sensitivity of the QQ-plots using the ShapiroWilks and the Kolmogorov-Smornov test, we show that;

$$
\sqrt{N}\left(\hat{\beta}_{m l e}-\beta\right) \rightarrow N\left(0, \frac{1}{I\left(\beta_{m l e}\right)}\right)
$$

where

$$
I(\beta)=-E_{\beta}\left(\begin{array}{llllll}
\frac{\partial^{2} \log l}{\partial \beta_{0}^{2}} & \frac{\partial \log l}{\partial \beta_{0} \partial \beta_{1}} & \frac{\partial^{2} \log l}{\partial \beta_{0} \partial \beta_{1}} & \frac{\partial \log l}{\partial \beta_{0} \partial \beta_{2}} & \frac{\partial \log l}{\partial \beta_{0} \partial \beta_{1}} \\
\frac{\partial^{2} \log l}{\partial \beta_{1} \partial \beta_{0}} & \frac{\partial \log l}{\partial \beta_{1}^{2}} & \frac{\partial^{2} \log l}{\partial \beta_{1} \partial \beta_{2}} & \frac{\partial \log l}{\partial \beta_{1} \partial \beta_{3}} & \frac{\partial \log l}{\partial \beta_{1} \partial \beta_{4}} \\
\frac{\partial^{2} \log l}{\partial \beta_{2} \partial \beta_{0}} & \frac{\partial \log l}{\partial \beta_{2} \partial \beta_{1}} & \frac{\partial \log l}{\partial \beta_{2}^{2}} & \frac{\partial \log l}{\partial \beta_{2} \partial \beta_{3}} & \frac{\partial \log l}{\partial \beta_{2} \partial \beta_{4}} \\
\frac{\partial^{2} \log l}{\partial \beta_{3} \partial \beta_{0}} & \frac{\partial \log l}{\partial \beta_{3} \partial \beta_{1}} & \frac{\partial \log l}{\partial \beta_{3} \partial \beta_{2}} & \frac{\partial \log l}{\partial \beta_{3}^{2}} & \frac{\partial \log l}{\partial \beta_{3} \partial \beta_{4}} \\
\frac{\partial^{2} \log l}{\partial \beta_{4} \partial \beta_{0}} & \frac{\partial^{2} \log l}{\partial \beta_{4} \partial \beta_{1}} & \frac{\partial^{2} \log l}{\partial \beta_{4} \partial \beta_{2}} & \frac{\partial \log l}{\partial \beta_{4} \partial \beta_{3}} & \frac{\partial \log l}{\partial \beta_{4}^{2}}
\end{array}\right)
$$

For different sample sizes of $n=500,700,1000,1500$, and2000, we calculate the Equation 11 and repeat it 5,000 times. The results are presented in the Figures 1, 2, 3, 4 and 5, through the quantile-quantile normal plot for $\hat{\beta}$.

A quantile-quantile normal graph, plots the quantiles of the data set against the theoretical quantiles of the standard normal distribution. If the data set appears to be a sample from a normal population, then the points will fall roughly along the line. The computation results indicates that the distribution of parameters approximates normal distribution as sample size, $n$ increases. 

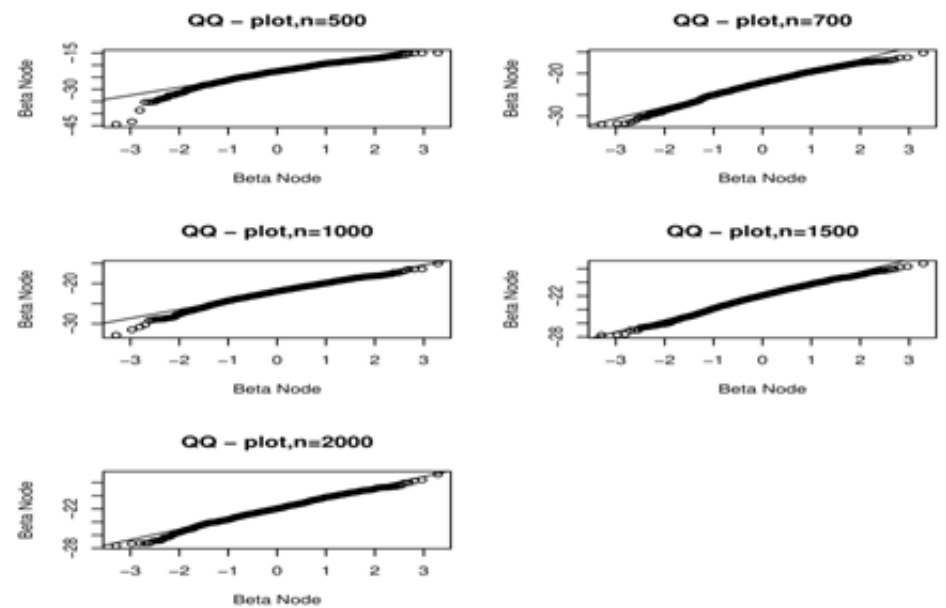

Figure 2: Monte Carlo Simulation of finite sample behaviour for normality of the parameter $\hat{\beta}_{0}$

Table 2: Test for Nomality $\beta_{0}$

\begin{tabular}{|c|c|c|c|c|}
\hline & Kolmogorov-Smir & test & Shapiro-Wilks test & \\
\hline Sample size(n) & statistic (D) $^{\text {Test }}$ & P-value & statistic (D) $^{\text {Test }}$ & P-value \\
\hline 500 & 0.0501 & 0.09864 & 0.9948 & 0.0016 \\
\hline 700 & 0.0664 & 0.0100 & 0.9961 & 0.0119 \\
\hline 1000 & 0.0389 & 0.3246 & 0.9964 & 0.01997 \\
\hline 1500 & 0.0325 & 0.5600 & 0.9987 & 0.3417 \\
\hline 2000 & 0.0323 & 0.5567 & 0.9986 & 0.0462 \\
\hline
\end{tabular}
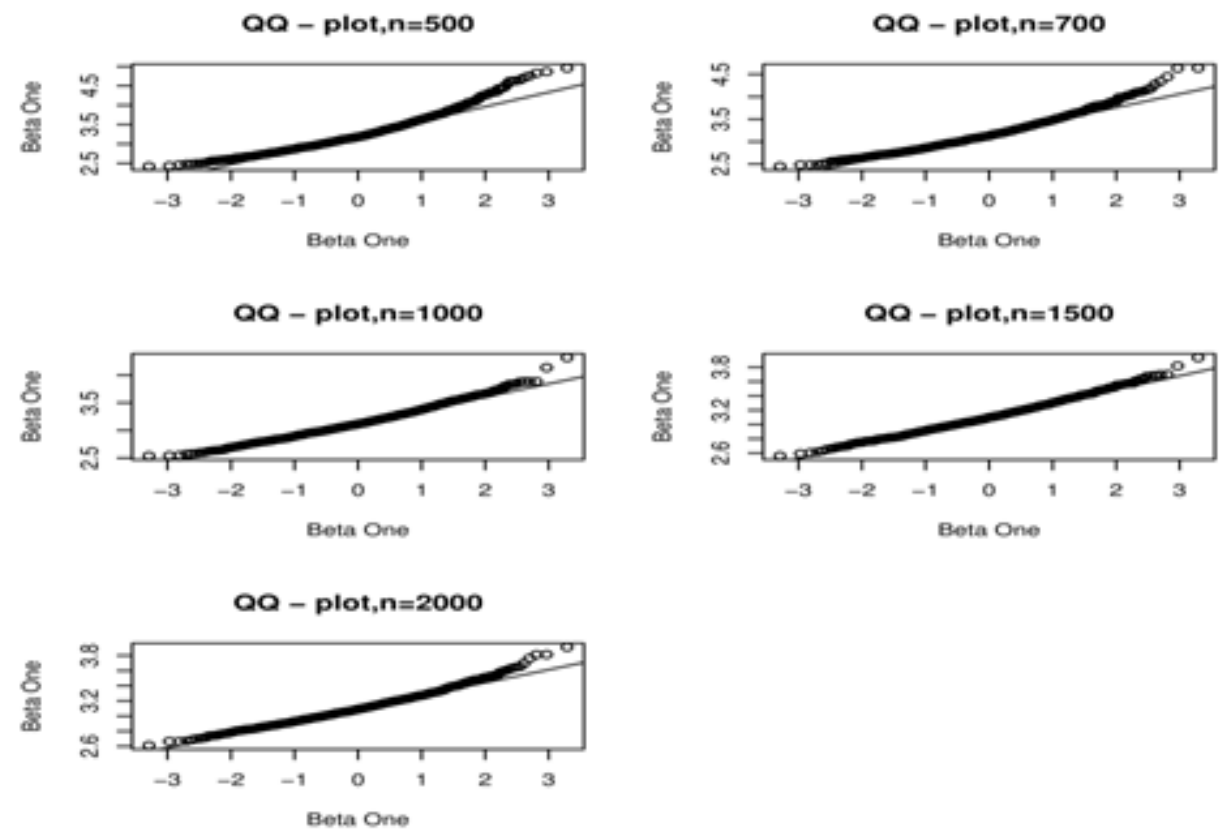

Figure 3: Monte Carlo Simulation of finite sample behaviour for normality of the parameter $\hat{\beta}_{1}$ Table 3: Test for Nomality

\begin{tabular}{ccccc}
\hline \multicolumn{3}{c}{ Kolmogorov-Smirnov test } & \multicolumn{2}{c}{ Shapiro-Wilks test } \\
\hline Sample size(n) & \multicolumn{2}{c}{ Test } & P-value & Test \\
& statistic (D) & 0.0015 & 0.0600 & P-value \\
\hline 500 & 0.0600 & 0.0037 & 0.0654 & 0.0015 \\
700 & 0.0584 & 0.0156 & 0.0493 & 0.0004 \\
1000 & 0.0493 & 0.0491 & 0.0431 & 0.0156 \\
1500 & 0.0431 & 0.2846 & 0.0312 & 0.0491 \\
2000 & 0.0312 & & & 0.2846 \\
\hline
\end{tabular}



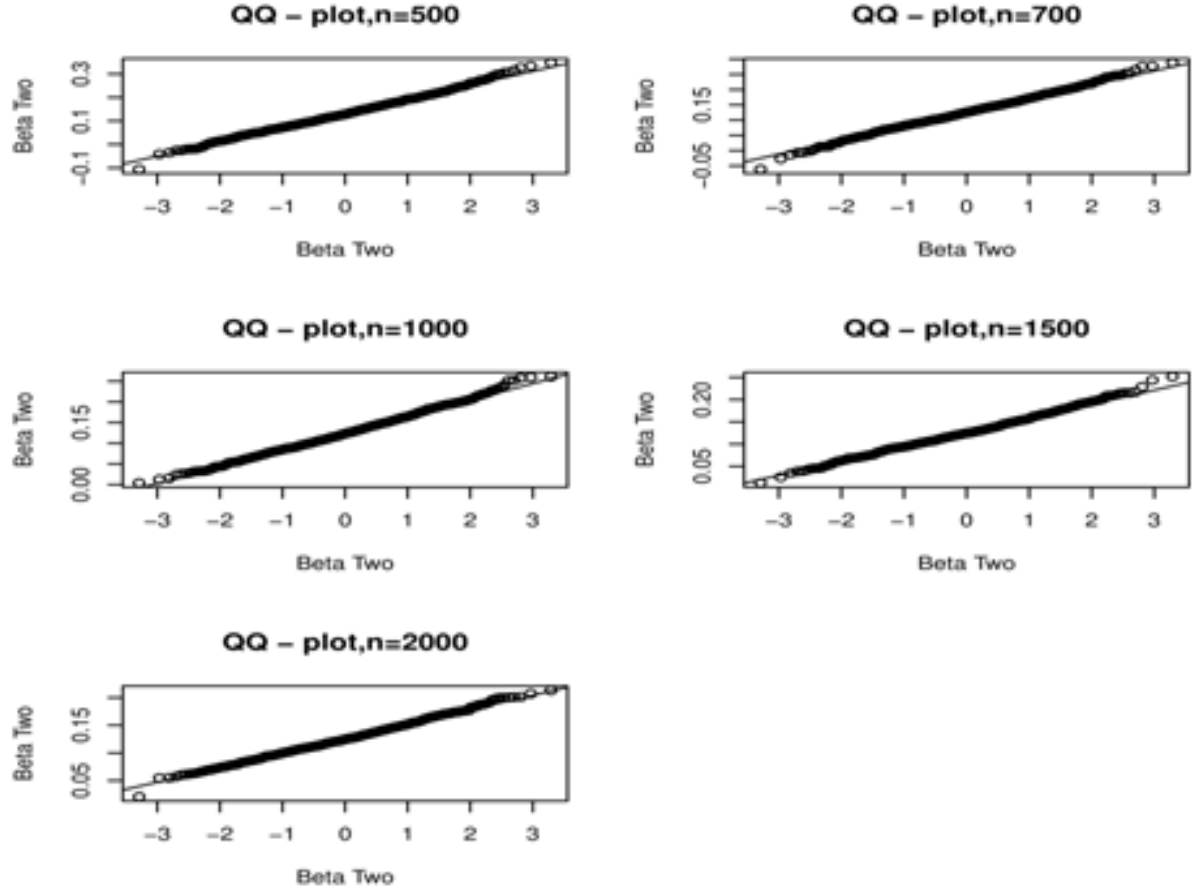

Figure 4: Monte Carlo Simulation of finite sample behaviour for normality of the parameter $\hat{\beta}_{2}$

Table 4: Test for Nomality

\begin{tabular}{ccccc}
\hline \multicolumn{2}{c}{ Kolmogorov-Smirnov test } & \multicolumn{2}{c}{ Shapiro-Wilks test } \\
\hline Sample size(n) & $\begin{array}{c}\text { Test } \\
\text { statistic (D) }\end{array}$ & P-value & Test & statistic (D) \\
\hline 500 & 0.0309 & 0.2948 & 0.9998 & 0.0899 \\
700 & 0.0346 & 0.1831 & 0.9970 & 0.1934 \\
1000 & 0.0326 & 0.2378 & 0.9961 & 0.05678 \\
1500 & 0.0295 & 0.3457 & 0.9974 & 0.0403 \\
2000 & 0.0291 & 0.3661 & 0.9995 & 0.1101 \\
\hline
\end{tabular}

$Q Q$ - plot, $n=500$

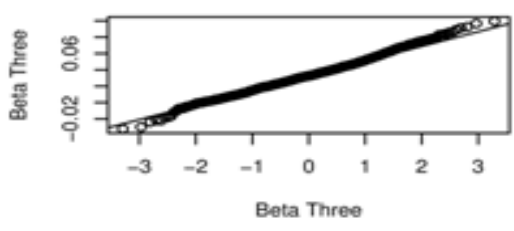

$Q Q$ - plot, $n=700$

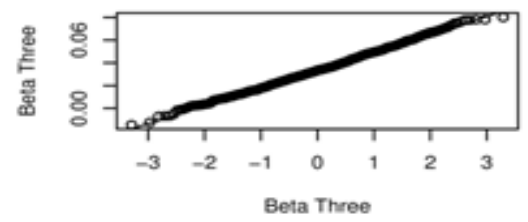

$Q Q-$ plot, $n=1000$

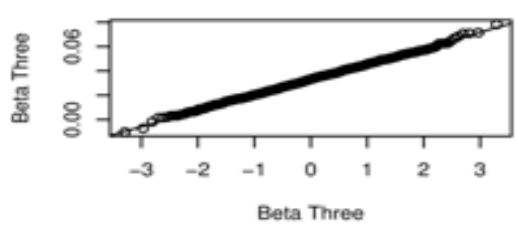

$Q Q-$ plot, $n=1500$

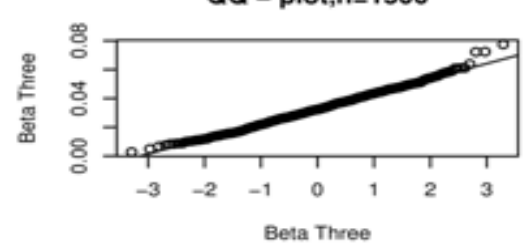

$Q Q-$ plot, $n=2000$

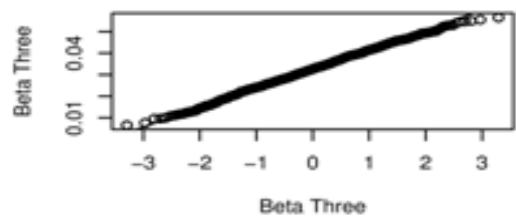

Figure 5: Monte Carlo Simulation of finite sample behaviour for normality of the parameter $\hat{\beta}_{3}$ 
Table 5: Test for Nomality

\begin{tabular}{|c|c|c|c|c|}
\hline & Kolmogorov-Smirnov test & & Shapiro- & \\
\hline Sample size(n) & $\begin{array}{l}\text { Test } \\
\text { statistic (D) }\end{array}$ & P-value & Test & P-value \\
\hline 500 & 0.0315 & 0.2731 & 0.9930 & 0.0001 \\
\hline 700 & 0.0287 & 0.3825 & 0.9952 & 0.0029 \\
\hline 1000 & 0.0167 & 0.5498 & 0.9969 & 0.0471 \\
\hline 1500 & 0.0122 & 0.8700 & 0.9945 & 0.0707 \\
\hline 2000 & 0.0096 & 0.8374 & 0.9988 & 0.7674 \\
\hline
\end{tabular}

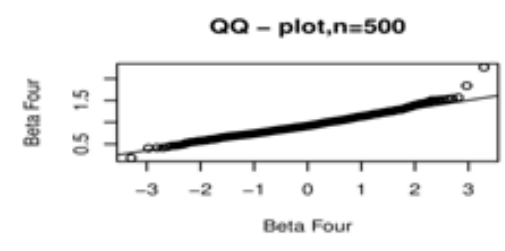

QQ - plot, $n=1000$

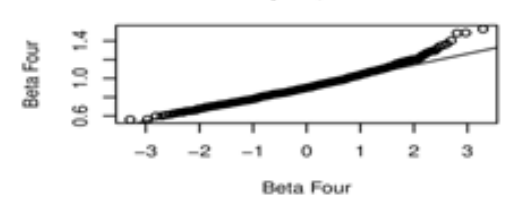

$Q Q-$ plot, $n=2000$

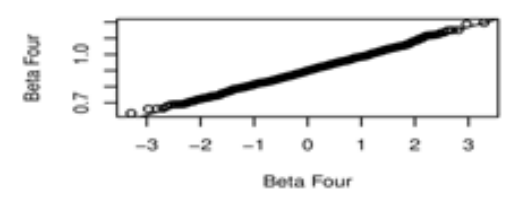

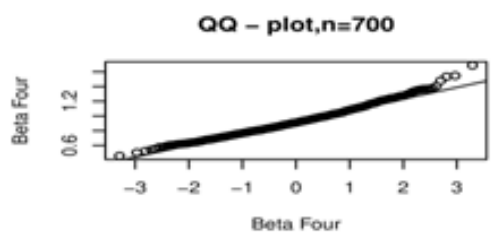

$Q Q-$ plot, $n=1500$

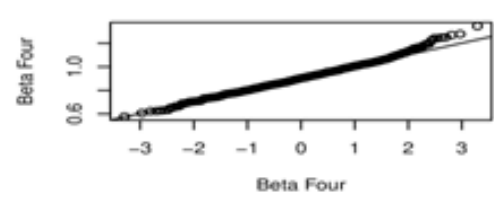

Figure 6: Monte Carlo Simulation of finite sample behaviour for normality of the parameter $\hat{\beta}_{4}$

Table 6: Test for Nomality

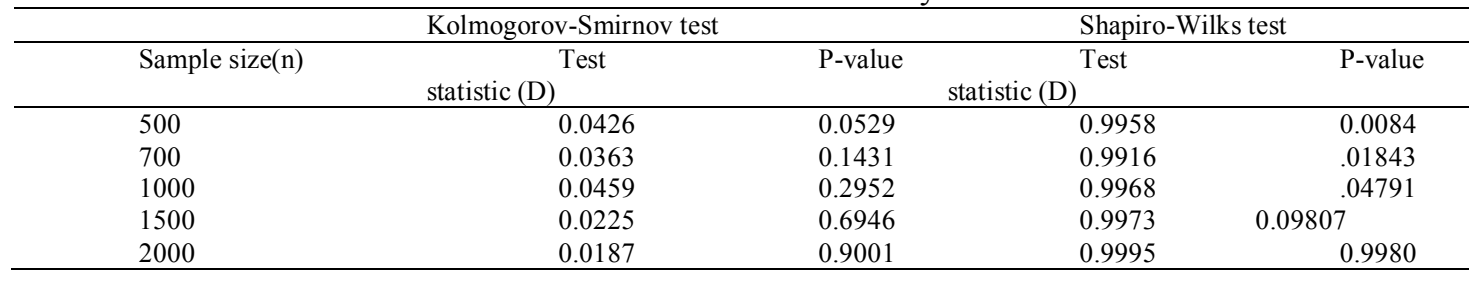

\section{Conclusion}

The study shows that the asymptotic properies of the maximum likelihood estimates of the logistic regression model can be obtained by some transformation of the regularity conditions of the linear regression model. The simulation studies done show that there is consistency in the parameter estimates, where fixed values of regression parameters are used, this shows that simulated estimates converge well to the fixed values as the sample size approaches infinity. The finite behaviour of consistency is upheld.

On the otherhand, simulated result on the normality were taken using the Q-Q-plots and using the the Kolmogorov-Smirnov and Shapiro-Wilks test. The analysis shows that the parameters are normally distributed, this can be checked on the decrease of the statistic values on both tests and also from tables 1, 2, 3, 4 and 5, we see that we fail to reject the null hypothesis at $\alpha=5 \%$ as the sample size increases and conclude that the samples are taken from the normal distribution.

\section{References}

[1] T. Amemiya. Advanced Econometric. Havard University Press, Cambridge, 1985.

[2] M. Beer. Asymptotic properies of the maximum likelihood estimator in [1] dichotomodi logistic regression model. 2001.

[3] David Collett. Modelling Binary Data. Chapman \& Hall/CRC, New York, USA, second edition, (2002).

[4] Jerome Cornfield. Joint dependence of risk of coronary heart disease on serum cholesterol and systolic blood pressure: A discriminant function analysis. In Federation Proceedings, volume 21, page 58, 1962.

[5] D. R. Sir Cox and E. J. Snell. Analysis of Binary Data. London.Chapman \& Hall, 1989.

[6] J. S. Cramer. Logit Models from Economics and Other Fields. Cambridge University Press, 2003.

[7] K. S. Crump, H. A. Guess, and K. I. Deal. Confidence intervals and test of hypothesis concerning dose response relations inferense. 1977. 
[8] R. C. Deutsch. Phd thesis. 2007.

[9] I. Fahrmeir and H. Kaufmann. Consistency and asymptotic normality of maximum likelihood estimator in generalized linear models. 1985.

[10] J. Fan, M. Farmen, and J. Gijbels. Local maximum likelihood estimator and inference. 1998.

[11] C. Gourienx and A. Monfort. Asymptotic properties of the maximum likelihood estimation in dichotomous logit models. Journal of Econometric 17, 83-97, 1981.

[12] H. A. Guess and K. S. Crump. Maximum likelihood estimation of dose response models subject absolutely monotonic constraints. 1978.

[13] D. W Hosmer and S. Lemeshow. Applied Logistic Regression. New York, Wiley, 1989.

[14] Trina Hosmer, David Hosmer, and Lloyd Fisher. A comparison of the maximum likelihood and discriminant function estimators of the coefficients of the logistic regression model for mixed continuous and discrete variables. Communications in StatisticsSimulation and Computation, 12(1):23-43, 1983.

[15] D. N. Joanes. Reject inference applied to logistic regression for credit scoring. 1993.

[16] G.S Maddala. Limited dependent variables and quantitative variables. 1983.

[17] P. McCullagh and J. A. Nelder. Generalized linear models. 1989.

[18] L. McFadden. Conditional logit analysis of qualitative choice behaviour. 1974

[19] L. Nordberg. Asymptotic normality of maximum likelihood estimators based on independent unequal distributed observations in exponential family models. 1980.

[20] Leandro Pardo. Statistical inference based on divergence measures. CRC Press, 2005.

[21] R. Pastor-Barriuso, E. Guallar, and J. Coresh. Use of two-segmented logistic regression to estimate change of point in epidemological studies. 1998.

[22] R. Pastor-Barriuso and E. Guallar. Transition model for change point estimation on logistic regression. 2003.

[23] M. Rashid and N. Shifa. Consistency of the maximum likelihood estimator in logistic regression model:a different approach. 2009.

[24] R.Christensen. Log-Linear Models and Logistic Regression. Springer-Verlag Inc., New York, USA, 1997.

[25] J Wilde. A note on gmm estimator of probit models with endogenous regression. 2008. 